\title{
DEVELOPMENT OF C-BAND ACCELERATOR STRUCTURE WITH SMOOTH SHAPE COUPLERS
}

\author{
T. Sugimura, T. Kamitani, K. Yokoyama, T. Takatomi, K. Kakihara, M. Ikeda and S. Ohsawa \\ KEK, 1-1, Oho, Tsukuba, Ibaraki, Japan
}

\begin{abstract}
A new C-band accelerator structure for the SuperKEKB injector linac is under development. This structure corresponds to an upstream half part in a tandem connection of two structures. A measure against RF breakdown is required because the upstream structure is exposed to higher RF field than that of the downstream structure. In order to suppress a RF breakdown around a coupler iris, a fat and smooth iris is adopted for the structure. The coupler dimensions such as an aperture of the iris and a diameter of the coupler cavity is first optimized by computer simulation and then decided by cold model measurements. Electric field symmetry is retained by shifting the coupler cavity from a beam centre. An amount of offset is also decided by simulations. The coupler body is made with a milling machine. A rather rough surface of the inner coupler is finished by an electro-polishing method. This structure will be installed in the beam line of the KEKB injector linac this summer.
\end{abstract}

\section{INTRODUCTION}

The next generation B-factory "SuperKEKB" project whose target luminosity is $1-5 \times 10^{35} \mathrm{~cm}^{-2} \mathrm{~s}^{-1}$ is under consideration [1]. Compared to the present KEKB the energy of an electron beam is lowered from $8 \mathrm{GeV}$ to 3.5 $\mathrm{GeV}$, and the energy of a positron beam is raised from 3.5 $\mathrm{GeV}$ to $8 \mathrm{GeV}$. The energy exchange of beams in order to escape an influence of electron clouds from photoelectrons has an important role. In the present KEKB injector linac, a positron is generated as a secondary particle on a tungsten target and then accelerated to $3.5 \mathrm{GeV}$. The accelerator length for a positron beam is sufficient for $3.5 \mathrm{GeV}$ but not for $8 \mathrm{GeV}$. One of solutions is to double an acceleration field gradient. Thus the Cband accelerator module which has double acceleration field gradient has been developed [2].

In the summer of 2003, the first accelerating structure (CKK001) was installed into the beam line and beam acceleration has been performed since. Attained beam accelerating field is $43 \mathrm{MV} / \mathrm{m}$ which satisfies our target value $42 \mathrm{MV} / \mathrm{m}$. As for the RF break down, frequency reduced to few times per day. It took about 1.5 years for RF conditioning. Most of the break downs occur in the input coupler. Thus the modification of the input coupler has been the subject of investigation for a middle term. It is assumed that the RF break down is caused by a thin iris, a difference in the level of a waveguide at the iris, and a sharp edge of the iris. To improve these points, a new design of the coupler is necessarily.

\section{MANUFACTURING STRATEGY}

The first accelerating structure is almost a half size of the accelerating structure for S-band. Methods of manufacturing are almost same. In an attempt to reduce a development term and get a fast start for an actual running test. There has been little effort to improve a coupler structure.

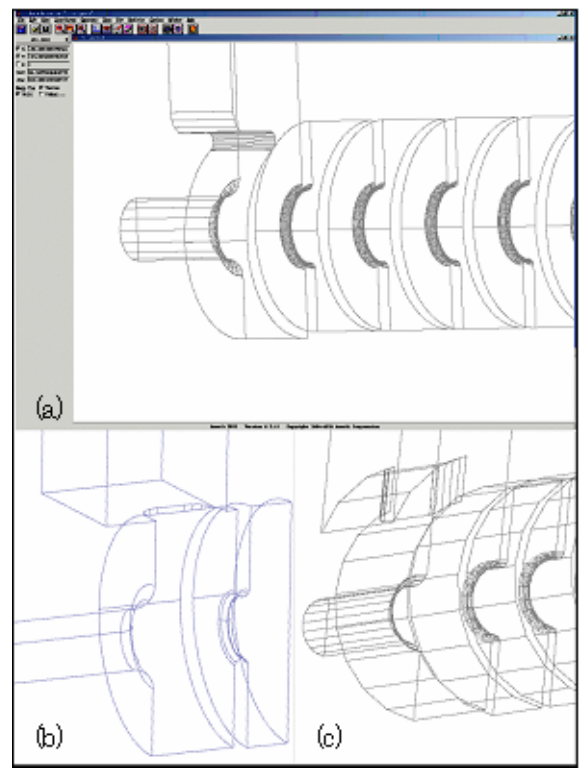

Figure 1: Wire-frame models of couplers for HFSS simulation. (a) The third model (CKK002), (b) The first model (CKK001), (c) The second model (CKK001)

The second structure (CKK001) has another type of couplers. One purpose of this coupler is to fatten the iris and another purpose is to test a step-less structure at the iris. This second type coupler renders coupler tuning with low power RF very difficult because a phase advance in the coupler cavity is not same as the regular cells $(2 \pi / 3)$ (see Fig.1 (c)). We almost gave up an edge-less iris for this coupler with the difficulties of processing except for filing down by hand $[3,4]$.

The third type coupler, which is our main concern in this paper, has a smooth shape from a waveguide to the coupler cavity in addition to a fat and step-less iris. Figure 1 (a) shows the half cutting model of the coupler which is used for a simulation described later. A phase advance in the coupler cavity returns to $2 \pi / 3$. The coupler is first processed by a milling cutter from a copper block, then parts are electro-polished, finally a side plate is attached to a coupler body by silver brazing. Since the third coupler is processed by a milling cutter in the coupler 
cavity, the inner surface of the cavity seems to be rather harsh compared to the surface of the first and the second ones processed by a lathe. This is the reason for introducing an electro-polishing method to a normalconducting cavity.
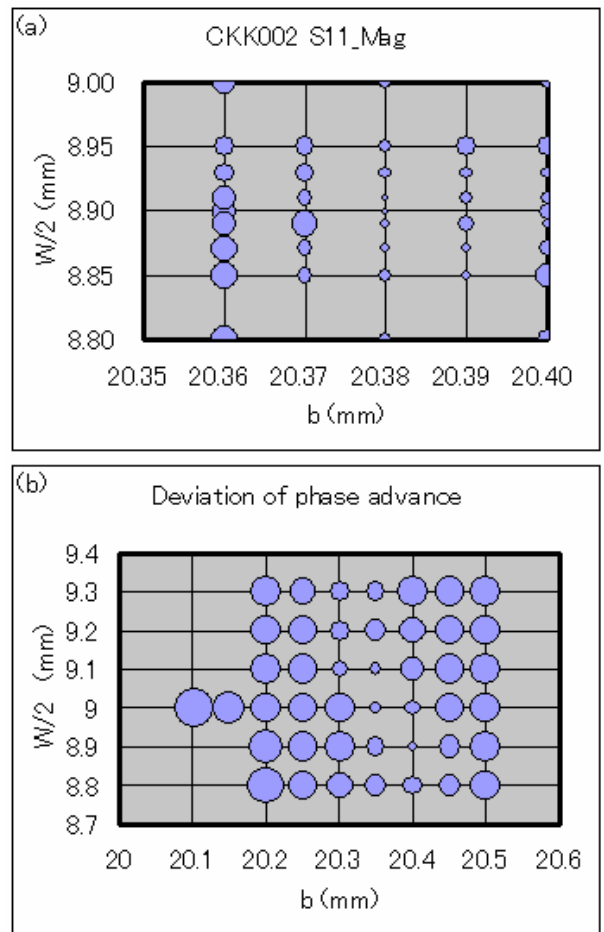

Figure 2: Results of HFSS simulations (a) A scattering matrix element $S_{11}$ is shown as a diameter of a circle. (b) A diameter of circle represents a summation of deviation of a phase advance from $2 \pi / 3$.

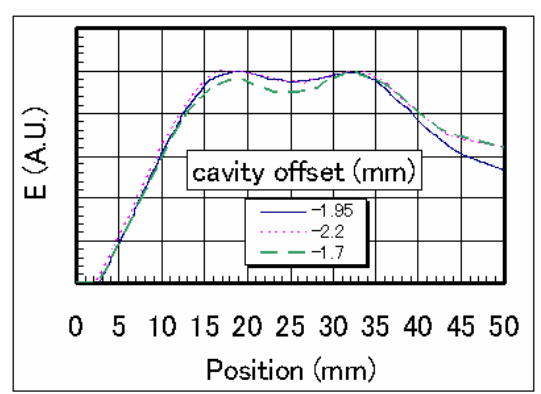

Figure 3: Electric field in the coupler with various offsets of coupler. Horizontal axis shows a position along the centre of the input waveguide. A position of $25 \mathrm{~mm}$ corresponds to the beam centre and the iris position is around $43 \mathrm{~mm}$ which depends on the offset of the coupler cavity.

\section{SIMULATION}

Coupler dimensions such as a diameter of a coupler (2b) and iris width (W) are determined by the Ansoft HFSS 8.5 simulation. There are two simulation models to optimize dimensions. One is a model with the input and output cavities putting a regular cell between them. In this model a scattering matrix element $\mathrm{S}_{11}$ is main concern. The other model simulates a nodal-shift measurement. In this model nodal-shifts of first 7 cells are simulated. A deviation of a phase advance in each cell from $2 \pi / 3$ is main interest. Figure 2 shows the simulation results. The diameters of circles represent $S_{11}$ or the summations of absolute value of the deviations. From results of simulations target values of $b$ and $W / 2$ for the cold model were decided to be $20.380 \mathrm{~mm}$ and $8.9 \mathrm{~mm}$, respectively.

An electric field in the coupler cavity is distorted by the iris. Symmetry of accelerating field on the beam axis is retained by slightly shifting the coupler cavity opposite to the iris. Amount of the offset is also simulated as shown in Fig.3. The offset of $-1.95 \mathrm{~mm}$ is selected for the cavity.

\section{COLD MODEL}

In order to determine the dimensions of the coupler, cold models were manufactured and RF measurements were performed. Six coupler bodies combining three size of $\mathrm{W}$ and two sizes of $2 \mathrm{~b}$ and seven beam-hole plungers with different radiuses of beam-hole corner (R) were prepared. Enlarging the radius has a same effect as reducing $2 \mathrm{~b}$ on the resonant frequency of the coupler cavity. Thus the radius is used as an adjustable parameter not only for cold models but also for high-power models. The RF measurements were performed by a Kyhl method and a nodal-shift method.

Fortunately one of combinations of a coupler body and a plunger has good RF properties, coupler dimensions are determined without interpolating. Figure 4 shows the result of the nodal-shift measurement for the coupler dimensions of $b=20.33 \mathrm{~mm}, \mathrm{~W} / 2=8.9 \mathrm{~mm}$ and $\mathrm{R}=3.5 \mathrm{~mm}$.

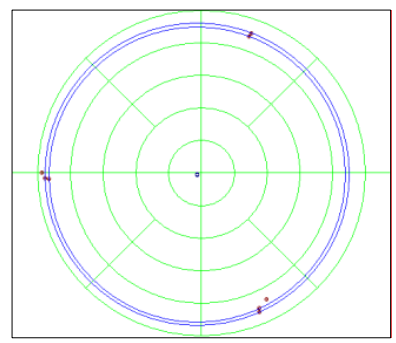

Figure 4: Results of nodal-shift measurement for cold model plotted on the polar chart: Measured points are indicated as red markers. A phase advance of each cell is in good agreement with $2 \pi / 3$. The circumscribed circle centre near the origin of chart indicates that reflection from cavity is quite small.

\section{HIGH POWER COUPLER}

Dimensions of the high-power coupler are based on the cold model. The high-power coupler cavity is processed by a milling cutter. In order to flatten the cutter marks on the surface of the inner cavity, electro-polishing about the depth of $30 \mu \mathrm{m}$ is applied. Figure 5 shows the couplers before and after electro-polishing. The cutter marks are disappointed clearly. Actually final dimensions are 
unknown because we can not touch the finished surface. A deviation of the electro-polishing has much effect on $2 b$ rather than $\mathrm{W}$. When the resonance frequency of the cavity is different from the target frequency by the effect of the deviation about $2 b$ it will be adjusted by the radius of the beam-hole corner before the final welding process of the beam-hole plunger. The deviation of $\mathrm{W}$ around 30 $\mu \mathrm{m}$ is considered to be a permissible range.

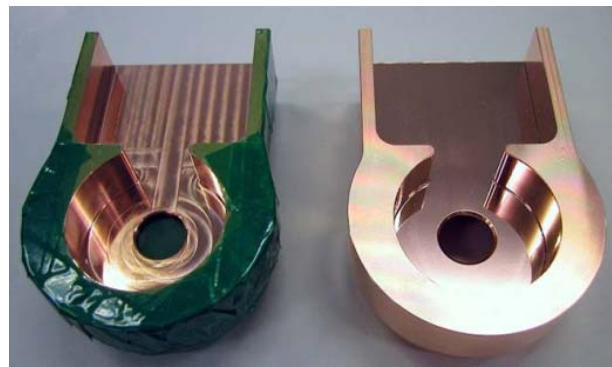

Figure 5: Effect of electro-polishing: Left and right coupler bodies are before and after electro-polishing, respectively. The cutter marks are disappeared by polishing.

\section{REGULAR CELLS}

Regular cells are constant impedance structure. One of merits of the structure is dimensions of input and output couplers are same. This means a development term is shortened. The aperture of the disk is $14.5 \mathrm{~mm}$ which imply that the group velocity in the structure is about $3 \%$ of the speed of light. This structure will locates on the upstream of the first or second type structure. This is the first time to process disks for upstream structure but there is no essential difficulty in processing because the way of manufacturing of this accelerating section is quite same as the second one. The inner diameter of spacers is defined by low power measurements using standard cavities. Surfaces of inner spacers are shaved with a lathe. Spacers are shaved in two or three steps. An aperture of spacers is first finished $20 \mu \mathrm{m}$ smaller than the target value, then about the half amount of compensation obtained from RF measurements is shaved, finally spacers are finished after checking that the frequency shift is as expected. Finished couplers, disks and spacers will be united by an electroplating about $4 \mathrm{~mm}$ in thickness. An electroplated structure is so hard that it can be used without tuning like a dimpling if all the regular cells are well tuned in advance.

\section{TOWARD THIS SUMMER}

\section{Electroplating}

The electroplating of the structure will start late in May. It takes about 2 weeks. For the S-band structure we adopt a fast electroplating method within a week for mass production. In a mass-production process, resonancefrequency shift caused by the stress from electroplating can be predicted and compensated in advance; whereas in trial manufacture it contains much unknown factors. Thus we adopt a slow electroplating method in order to reduce a frequency shift caused by the stress, which falls down as current density of electroplating falls down. This slow electroplating method was also adopted for the second structure.

\section{Welding water jackets and flanges}

The structure will be covered with cooling water jackets. The water jackets, flange and the structure are TIG welded each other. The coupler cell has a tendency to be deformed by welding, thus a temperature must be well controlled during a welding process.

\section{Welding beam-hole plungers}

Though welding a beam-hole plunger is the final process of manufacturing a structure, a resonance frequency of the coupler cavity changes during the welding process. The amount of the frequency shift will be estimated by the measurement of another test coupler. Compensation of the frequency shift will be reflected as a radius of the beam-hole corner. A temperature control is also necessary in this welding process.

\section{$R F$ processing}

Before installation in the beam line of the injector linac, high-power RF processing will be performed at a test stand. From the restriction of our schedule, this structure and another one are processed at the same time. It will take about 2 weeks to complete.

\section{SUMMARY}

A new C-band accelerator structure with a smooth shape coupler is now under development. The coupler iris is fat, step-less and edge-less. Decisions of coupler dimensions are based on the simulations and the cold model measurements. An electro-polishing method is newly introduced to the coupler inner surface. The structure will be completed and installed in the beam line of the KEKB injector linac in this summer.

\section{ACKNOWLEDGEMENT}

The authors would like to give their sincere thanks to Mr. Y. Ino at Mitsubishi Heavy Industries, LTD. for his cooperation.

\section{REFERENCES}

[1] J. W. Flanagan, Y. Ohnishi, et al., Letter of Intent for KEK Super B Factory, Part III: Accelerator Design, KEK Report 04-4

[2] T. Kamitani, et al., "Development of C-band accelerating section for SuperKEKB", Proc.of LINAC 2004, Lübeck, Germany, Aug, 2004, pp663

[3] T. Kamitani, et al.,"R and D Status of C-Band Accelerating Section for SuperKEKB", this conference

[4] K. Yokoyama, et al., "Coupler Matching Techniques for C-Band Accelerating Section”, this conference 